\title{
The Influence of Stratified Training with Post Obligation as the Core on the Comprehensive Ability of Junior Nurses
}

\author{
Liangfeng Yang ${ }^{1}$, Xiaofeng Li ${ }^{2}$,, Zuxi Feng ${ }^{3}$, Lu Tan $^{2}$, Lei Chen ${ }^{4}$, Ruyue Liu ${ }^{5}$ \\ ${ }^{1}$ The Department of Quality Management, Second People's Hospital of Three Gorges University, Yichang Second People's Hospital, Yichang, \\ China \\ ${ }^{2}$ The Department of Nursing, Second People's Hospital of Three Gorges University, Yichang Second People's Hospital, Yichang, China \\ ${ }^{3}$ Chairman of the Labor union, Second People's Hospital of Three Gorges University, Yichang Second People's Hospital, Yichang, China \\ ${ }^{4}$ Pharmacy Intravenous Admixture Service, Second People's Hospital of Three Gorges University, Yichang Second People's Hospital, Yichang, \\ China \\ ${ }^{5}$ Emergency Department, Second People's Hospital of Three Gorges University, Yichang Second People's Hospital, Yichang, China
}

\section{Email address: \\ 359377184@qq.com (Xiaofeng Li) \\ ${ }^{*}$ Corresponding author}

\section{To cite this article:}

Liangfeng Yang, Xiaofeng Li, Zuxi Feng, Lu Tan, Lei Chen, Ruyue Liu. The Influence of Stratified Training with Post Obligation as the Core on the Comprehensive Ability of Junior Nurses. American Journal of Nursing Science. Vol. 8, No. 3, 2019, pp. 104-108.

doi: 10.11648/j.ajns.20190803.15

Received: February 26, 2019; Accepted: April 11, 2019; Published: May 9, 2019

\begin{abstract}
Objective: To explore the effect of stratified training with post obligation as the core to improve the comprehensive ability of junior nurses. Methods: 565 nurses with low seniority were divided into two groups: observation group and control group. 283 participants adopted the hierarchical training model with post obligation as the core.To determine the post responsibilities of nursing staff with low seniority on the basis of post setting and post access. According to the job responsibilities of nurses with low seniority to determine the training content of nurses with low seniority, refined comprehensive quantitative assessment standards. The assessment is taken as an important basis to test the training effect and the completion of the post responsibilities of the nursing staff with low seniority. Form a trinity of job responsibilities, training content and assessment standards training mode. The training effects of the two groups were compared. Results: The evaluation results, patient satisfaction and training satisfaction of nurses in observation group were all higher than those in control group. There were statistically significant differences between the two groups (all p \&lt; 0.05). Conclusion: Stratified training based on post obligation as the core can combine the post demand of junior nurses, improve the learning enthusiasm and post competence, and thus improve the quality of service.
\end{abstract}

Keywords: Post Obligations, Junior Nurses, Hierarchical Training

\section{Introduction}

Nursing staff of different levels have different post difficulty and comprehensive ability. Nurses with low seniority are the new force in the nursing team. Compared with senior nurses, their abilities to observe, communicate and solve practical problems with theoretical knowledge are relatively weak [1]. Therefore, it is an important task to improve the nursing quality, patient safety and nursing personnel training to improve the comprehensive ability of low seniority nurses in light of actual needs [2]. Under the premise of promoting post access and hierarchical use of nursing staff, this project carries out trainings focusing on post obligations in combination with the characteristics and post demands of nursing staff with low seniority, with good results. The report is as follows:

\section{Data and Methods}

\subsection{General Information}

A total of 565 junior nurses in the hospital from January to December 2018 were randomly divided into the observation 
group and the control group. In the control group, 282 subjects received traditional theoretical operation training. In the observation group, 283 participants adopted the hierarchical training mode with post obligation as the core. Excluded objects: nursing personnel who have not obtained the nurse's professional qualification, have not completed the probationary training for new nurses, have been transferred from nursing posts and have rested for more than 2 months. There was no statistical difference between the two groups in age, gender, education background and professional title (all $\mathrm{P}>0.05)$.

\subsection{Method}

\subsubsection{The training Mode of Junior nursing Staff based on Post Obligation}

The observation group implemented the three-in-one hierarchical training mode of post responsibility, training content and assessment standard of nursing staff at all levels on the basis of post setting and post access. See figure 1 for the training pattern of junior nurses.

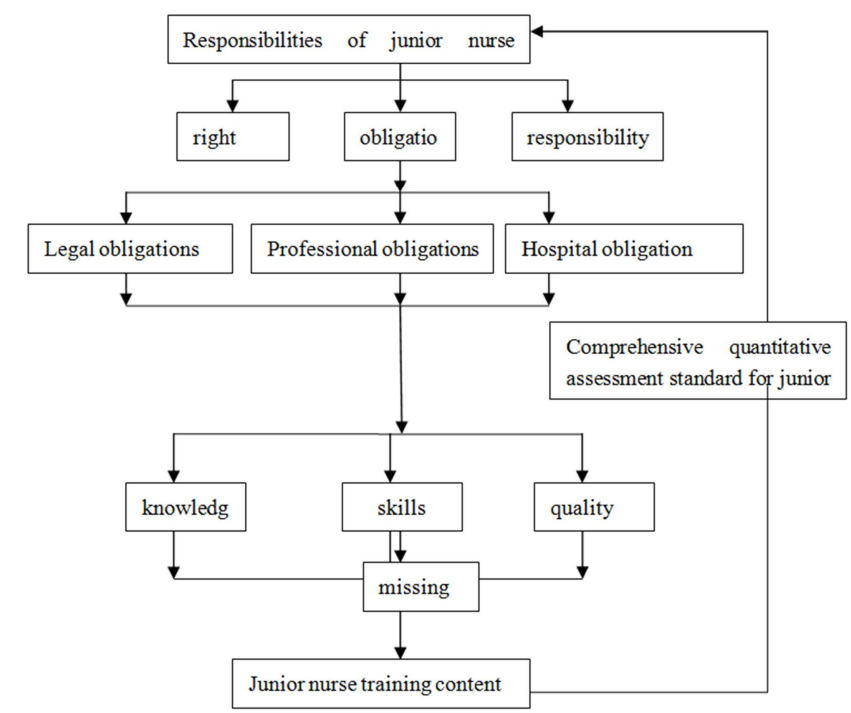

Figure 1. Schematic diagram of training mode for junior nurses with post obligation as the core.

\subsubsection{Stratification of Junior Nurses}

According to professional title, educational background, qualification, ability and position, nurses with low seniority divide clinical nursing staff into five levels. N0: new nurse, working time within 1 year, junior college degree; N1 : a nurse who has worked for 2-3 years, with a junior college degree, has obtained the professional qualification, and is qualified for general clinical nursing work; N2: a nurse who has worked for 4-10 years, with a junior college degree and the title of nursing teacher. She is competent for clinical nursing work with great difficulty. N3: 11-15 years of work experience, junior college degree, nursing supervisor or above title, with the ability to solve problems in the undergraduate nursing business; N4: a nurse who has worked for more than 16 years, with a bachelor's degree and a nursing teacher title of director (deputy director), with the ability to deal with difficult and severe diseases, teaching and scientific research. Among them, N0 and N1 are junior nurses.

\subsubsection{The Training Content}

On the basis of clarifying the post obligations of nurses at all levels, the training contents were determined through the survey of patient satisfaction, analysis of adverse events, pre-training assessment of nurses with low seniority and survey of training needs: Quality (interpersonal communication, practical manners, ethics, career planning and psychological adjustment), knowledge (the basic nursing theory and operation, the primary specialty theory and basic theory of operation, the new business and operation, patient safety assessment and prevention, key drugs related knowledge, hospital infection knowledge, laws and regulations, system process, computer), skills (application of nursing process, health education path, document writing, the hospital electronic information system, occupational safety protection, emergency planning process, adverse events prevention and reporting, the use and maintenance of specialized instruments) 3 categories. In the process of training organization, the training contents are summarized and analyzed. The common parts are organized by the nursing department, and the training of major departments and departments are further refined with the combination of specialties. During the training period, the performance of the post obligations should be adjusted in a timely manner.

\subsubsection{The Assessment Method}

A combination of short-term individual and long-term comprehensive assessment. Short-term individual assessment is the immediate evaluation of all kinds of theories, operations, new business training, such as the three basic three strict monthly assessment, the outstanding person given a single reward, non-conforming person retraining. The long-term comprehensive assessment is based on the "comprehensive quantitative assessment standards for low seniority nurses" to investigate the practical application of training content in clinical daily work. Finally, through self-evaluation, three-way quantitative scoring of major department and nursing department, the performance of the evaluation result is linked to the promotion, evaluation and performance of nursing staff.

I Comprehensive quantitative assessment indicators

There are 22 secondary assessment indicators under the three primary assessment indicators of quality, knowledge and skills. They are: quality (standard words and deeds, medical ethics, comprehensive awards, public welfare activities ) ; knowledge (basic theoretical operation, specialized theoretical operation, system mastery, multimedia office software application, professional awards); Skills: (task completion, quality of basic nursing, quality of specialized nursing, critical nursing for patients, implementation of core systems, emergency treatment, writing of documents, health guidance, safety prevention and adverse events, patient satisfaction, doctor satisfaction, training and teaching, summary and innovation). 
II Stratified determination of comprehensive quantitative assessment score and difficulty

The combination of qualitative and quantitative analysis, using the percentage weight analysis method, after expert group verification and approval. The determination of the score of each assessment index comprehensively considers the responsibility level to fulfill the post obligation, patients' demand for the nursing post obligation, professional difficulty and development and other factors. The score is in direct proportional to the degree of risk, technical difficulty, labor intensity and time spent. The evaluation content and difficulty degree of each index were adjusted according to the duty category and degree of nursing staff at all levels.

\section{Statistical Processing}

SPSS19.0 software was used, $\mathrm{x}^{2}$ test was used for counting data, and $t$ test was used for measuring data.

\section{Results}

\subsection{Comparison of Adverse Events Incidence and Satisfaction Between the Two Groups (See Table 1)}

Table 1. Comparison of incidence of adverse events and satisfaction between the two groups.

\begin{tabular}{lllll}
\hline Group & The number of & Adverse events (example) & Patient satisfaction (\%) & Nursing staff training satisfaction (\%) \\
\hline The control group & 282 & 12 & 91.18 & 84.17 \\
Observation group & 283 & 17 & 95.39 & 92.94 \\
$\mathrm{X}^{2}$ & & 0.89 & 4 & 10.73 \\
$\mathrm{P}$ & & $>0.05$ & $<0.05$ & $<0.05$ \\
\hline
\end{tabular}

\subsection{Comparison of Assessment Results Between the Two Groups of Nursing Staff (See Table 2)}

Table 2. Comparison of assessment results between the two groups of nursing staff $(\bar{x} \pm s)$.

\begin{tabular}{lllll}
\hline Group & $\begin{array}{l}\text { The number } \\
\text { of }\end{array}$ & $\begin{array}{l}\text { Basic knowledge and } \\
\text { skills }\end{array}$ & $\begin{array}{l}\text { Specialized knowledge } \\
\text { and skills }\end{array}$ & $\begin{array}{l}\text { Comprehensive quantitative } \\
\text { assessment results }\end{array}$ \\
\hline The control group & 282 & $73.5 \pm 6.73$ & $68.53 \pm 5.93$ & $78.69 \pm 3.69$ \\
Observation group & 283 & $89.1 \pm 5.64$ & $84.68 \pm 4.66$ & $85.17 \pm 3.46$ \\
$\mathrm{t}$ & & 29.87 & 36.00 & 16.93 \\
$\mathrm{P}$ & & $<0.05$ & $<0.05$ & $<0.05$ \\
\hline
\end{tabular}

\section{Discussion}

\subsection{The Importance of Training Junior Nurses}

At the beginning of 2010, the Ministry of Health required all levels of hospitals to actively carry out job training for clinical nurses, and conduct scientific, targeted and practical training based on job demand. At present, the post responsibilities of the hospital mainly include the minimum educational background, job qualifications, professional knowledge, work experience, work attitude, physical requirements, etc. [3]. Among them obligation is the core that fulfils post duty. The growth of nursing staff is a gradual process, combined with the characteristics of low-seniority nursing staff, job demand for training, which is of great significance to improve clinical nursing ability and career growth of nursing staff. This study compared the post obligations of low seniority nurses, and took legal obligations, professional obligations and hospital obligations as the basis for training and assessment. This three-in-one training model of job responsibilities, training content and assessment standards, with the same academic application, the right to clear responsibility, the goal of quantification, so that the low- seniority nurses can compare the standards to find the gap, know where the gap? What to learn? What to do? To what extent? It can effectively help low- seniority nurses to fulfill their responsibilities, and make clear how to approach higher-level positions through training, which is conducive to the development of nursing career planning and the echelon training of talent team [4].

\subsection{Training Model with Post Obligation as the Core to Improve Post Competency}

Using the acquired knowledge and skills to optimize nursing services [5] is an important goal of nursing training. Assessment is an important means to promote the transformation of training results into productivity [6], so nursing training and assessment are complementary and inseparable, and scientific assessment method can effectively improve the training effect [7]. Traditional training and assessment of theoretical knowledge and operational skills are difficult to objectively evaluate the comprehensive ability, humanistic cultivation and professional quality of nurses [8]. According to the requirements of knowledge, quality and skills of nursing staff at all levels, this model sets the comprehensive quantitative assessment standard for junior nurses by combining qualitative and quantitative methods. Can be the assessment indicators control data, standardization. It avoids the arbitrariness, one-sidedness and man-made factors of assessment [9]. Through the comprehensive quantitative assessment of the performance of the test post obligation, to promote the transformation of training results into nursing practice. Through the standardized stratified training and assessment, the junior nurses are engaged in the nursing work that matches their abilities and responsibilities. Avoid over grade and over capacity operation, improve nursing quality. In 
the survey of training needs, this study showed that junior nurses had the highest demand for basic theory and operation, common professional knowledge and skills, occupational safety protection, and communication between nurses and patients. The patient satisfaction questionnaire indicated the hope of improving the venipuncture level of junior nurses and skilled use of specialized instruments and equipment. Targeted training for the above contents can help them better complete daily practical work and reduce occupational pressure caused by patients' dissatisfaction. The common part of the training content is organized by the nursing department. Each department further refined the specialized training to meet the health needs of patients with different diseases and improve the quality of care. Table 2 showed that the short-term assessment results of basic knowledge and skills, specialized knowledge and skills of nurses in the observation group were significantly improved compared with the control group, and the long-term comprehensive quantitative assessment results of daily work were also significantly improved. Table 1 showed that the satisfaction of patients in the observation group is higher than that in the control group. Although there is no statistical difference in the number of adverse events between the observation group and the control group, further analysis showed that 17 adverse events in the observation group are all level 4 hidden danger events without causing errors or injuries. This is closely related to the improvement of nursing staff's initiative in adverse event reporting after training and their mastery of the reporting process.

\subsection{The Training Model with Post Obligation as the Core to Improve the Training Enthusiasm and Satisfaction of Nurses}

The annual comprehensive quantitative assessment results of this mode, as the performance of post obligations, have a continuous impact on promotion, evaluation and performance. Those who do not pass the annual comprehensive quantitative assessment can not participate in the first, promotion. Two consecutive years of failure to demote the use. Those who do not pass the annual comprehensive quantitative assessment can not participate in the first, promotion. Two consecutive years of failure to demote the use. Avoid training one thing, work another thing; The training and working enthusiasm of nursing staff should be maintained for a long time [10]. Different post levels, each comprehensive quantitative assessment indicators of the evaluation content, degree of difficulty is also different. For example, "training and teaching" focuses on the frequency and time requirements for junior nurses to participate in training; Senior nurses pay more attention to the assessment of the organization of continuing education, teaching quality and high-level academic exchanges. In "summary and innovation", N0 level only needs to learn clear and standard notes and write comments and experiences. N1 level needs to participate in papers, new business, patents and other related activities, while N4 level has higher requirements for papers, scientific research, patents and other levels and rankings. Table 1 showed that the observation group is significantly more satisfied with the training than the control group. Hierarchical training and assessment can reduce the training pressure of nursing staff and improve the training enthusiasm and satisfaction.

\section{Summary}

The " Outline of 2030 Planing for Healthy China" has put forward new requirements for the development of nursing, which should play a professional role in disease prevention, health promotion, diagnosis and rehabilitation, care and care in the whole process of life and death [11]. Combined with the outline of nursing training, it is also necessary to keep pace with the times and realize the training of application-oriented high-quality talents with Chinese characteristics that are close to the people [12]. However, the cultivation of a large nursing team cannot be accomplished overnight, and it is not enough to simply and blindly seek more and faster. It is necessary to grasp the key points, guide correctly and make gradual progress step by step. Therefore, the training of nurses with low seniority is an important basis to improve the quality of clinical nursing team. This training mode starts from the post demand, determines the training content and assessment standard, closely combines the connotation of nursing service with the health needs of the people, and improves the comprehensive ability and post competence from the aspects of knowledge, quality and skills, which is conducive to the formation of nurses' caring ability and positive values [13]. Thus constantly improve the quality of nursing, and also lay a more solid foundation for the development of nursing professional.

\section{Project}

This project is the Yichang science and technology bureau soft science project A14-30322 in 2014.

\section{References}

[1] Liping Xie, Xiaofeng Li, Liangfeng Yang. Application of "six heart" in operation training of junior nurses [J]. Nursing practice and research, 2016, 13(06): 131-132.

[2] Lei Chen, Liangfeng Yang. Application of hierarchical training mode with post obligation as the core in the training of low-seniority nurses in orthopedics department $[\mathrm{J}]$. Nursing of integrated traditional Chinese and western medicine (both Chinese and English), 2018, 4(02): 155-158.

[3] Zhu Qiqing, Gang Du, Weiguang Li. design and application of hospital job description [J]. Chinese journal of hospital management, 2003, $1: 739-740$. (in Chinese)

[4] Liangfeng Yang, Qingping Xiang, Xiaofeng Li, Yuanyi Tan. Current situation of hierarchical training and assessment system for nursing staff $[\mathrm{J}]$. Nursing practice and research, 2016, 13(02): 26-27. 
[5] Chang Wei, Jianrong Wang, Liming Zhang. Methods and effect analysis of hierarchical training and assessment for nurses [J]. Journal of nursing management, 2006, 6 (6) : 7-9.

[6] Lili Chen. Preliminary study on the application of hierarchical assessment of nursing staff [J]. Tianjin nursing, 2010,18 (3) : 154-155.

[7] Liangfeng Yang, Qingping Xiang. Research on the application of hierarchical training model with post obligation as the core [J]. Nursing research, 2015, 29(35): 4416-4418.

[8] Wenju Dong, Miaoqin Wang. Application of QSCE in the assessment system of general nursing training [J]. Journal of nursing, 2013, 28 (7) : 31-33.

[9] Hongzhu Wu. Application of comprehensive quantitative assessment in nursing management $[\mathrm{J}]$. Hospital management BBS, 2012, 29 (1) : 51-53

[10] Siping Hu. Preliminary study on on-the-job training of senior nurses in the new era $[\mathrm{J}]$. Journal of changjiang university, 2009, 6 (4) : 415-418.

[11] Yanhong Guo. Implementation of healthy China strategy to promote the development of nursing [J]. Chinese journal of nursing, 2008, 53(01): 5.

[12] Yuqian Zhou. A brief analysis of the cultivation of nursing master in the context of health China [J]. China health industry, 2008, 15(35): 190-191.

[13] Li Fan, Juan Li. Current situation and correlation research on core competence and caring ability of nurses with low seniority [J]. Shanghai nursing, 2008, 18(10): 79-82.

\section{Biography}

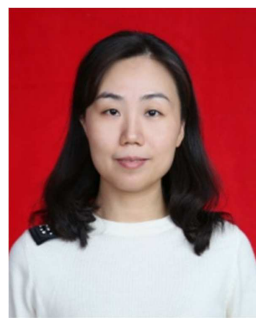

Liangfeng Yang (1979-) Yichang City, Hubei Province, China, Deputy director of quality management Department of the second people's hospital of three Gorges university in Yichang city, deputy director of nursing division, undergraduatedegree research direction: clinical nursing and nursing management.

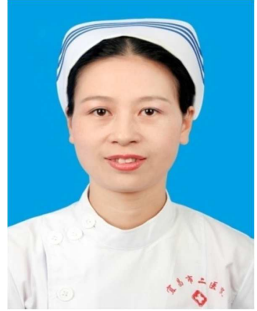

Xiaofeng Li (1969-), Yichang City, Hubei Province, China, director and chief nurse of the nursing department of Yichang Second People's Hospital, is a professor of the nursing department of Three Gorges University, editorial board member of journal of nursing science and other magazines, director of Hubei Nursing Association, standing member of Hubei Cancer Nursing Professional Committee, deputy director and secretary-general of Yichang Nursing Association, and chairman of Yichang Cancer Nursing Branch. She edited 5 nursing monographs, published more than 50 papers in core journals, presided over 5 scientific research topics, and won awards such as Hubei Provincial Progress Award. In April 2017, she was named the director of the national excellent nursing department. 